Session 13b2

\title{
Collaborative Undergraduate Research
}

\author{
Hamid Davoodi, Frederick Just \\ Mechanical Engineering Department \\ Ali Saffar \\ Civil Engineering Department \\ University of Puerto Rico at Mayaguez \\ Mayaguez, PR 00681 \\ Mohammad Noori, William Durgin \\ Mechanical Engineering Department \\ Worcester Polytechnic Institute \\ Worcester, MA 01609
}

\begin{abstract}
A joint undergraduate research project between Untversity of Puerto Rico at Mayaguez (UPRM) and Worcester Polytechnic Institute (WPI) started four years ago. Each year a group of students, varying between six and ten, form WPI will come to Puerto Rico to work with a group of student, varying from six to ten, from UPRM on a well defined research project. Although the topics of the projects have been different but all follow the same goal, Vibration Control of Siructures by Utilizing Shape Memory Alloys, Each year the project starts on Jamuary and ends on May. Both groups of the students will start the projeat simultaneously on their respective universty. Seven weeks after the stant of project, WPI students come to Puerto Rico and joint the UPRM group and contime their research as "one group." The requirements for completion of these projects are two major presentations, one at each university. and one written report. The presentations are all open to public, and students, faculy members, the local industry, and parents are all being invited. The projects have been sponsored by United Technology. Center for Loss Prevention and Siruciural Integrity (CLPSI) at WPI, WPI and UPRM. The results of these projects have been presented at CANAM 97 and $12^{\text {sh }}$ ASCE Conference. There have been also numerous published articles and interview related to these projects in engineering journals and televisions.

Worcester Polytechnic Institute (WPI) and the University of Puerto Rico (UPR) entered into a cooperative agreement in 1995. That agreement enabled broad areas of exchange, collaboration, and joint research.
\end{abstract}

\section{Background}

As the nation's thitd oldest private college of science and technology, Worcester Polytechnic Institute (WPI) is a pioneer in engineering education having developed a curriculum that balanced classroom instruction with practical, hands on experience in a real world settings. The engineering program at WPI has been structured around a project based, multidisciplinaty, team approach. This system has provided a unique infrastructure for responding to new challenges facing the engineering education, including societal, global, and outcomes concerns.

WPI's program requires all students to complete two significant projects to earn the baccalaureate degree. First, the Major Qualifying Project (MQP) must be in the student's major area of study and focuses on the demonstration of the skitls, method's, and knowledge of the discipline to solving a problem representative of the type to be encountered at the professional level. This team project is usually undertaken in student's senior year. Second, the Interactive Qualifying Project (IQP) challenges students to identify, investigate, analyze and reports on a problem at the interface of technology and society. Specifically, students develop an understanding of the ways in which technology is developed to meet societal needs and how now technologies affect our society.

The University of Puetto Rico at Mayagdez (UPRM) is a land grant institution. The campus consists of three colleges in the areas of Arts and Science, Agriculture and Engineering. UPRM provides over 20 percent of the Hispanic engineering work force in the United States. In addition, it contains the highest percentage of female students in science and engineering among all science and engineering schools in the U.S. The five-year engineering program at UPRM allows students to gain a broad background in all engineering fields. It also allows the students to participate on research projects under special courses. Through these courses the student gain the experience that is needed in pursuing advance degree before finishing their BS.

Both universities appreciate the importance of developing the global perspectives of engineering students. Morc than one-half of WPI's engineering students conduct some of their project work, whether 
IQP or $\mathrm{MQP}$, at a residential site. These project centers are located throughout the world and are designed to provide opportunity for students to conduct professional level work in an unfamiliar culture. UPRM has long had emphasis on developing global perspectives through exchange programs, faculty development programs, and a willingness to collaborate with the universities in educational research.

\section{Institutional collaboration}

In Nov-ember of 1995 a memorandum of agreement was signed between WPI and UPRM. One of the elements of this agreement was "undergraduate rescarch". Each year a group of students, varying between six and ten, from WPI will come to Puerto Rico to work with a group of students, varying from six to ten, from UPRM on a well defined research project. The project starts on January and ends in May each year. WPI and UPRM students start the project simultaneously at their respective university. After seven weeks, WPI students travel to Puerto Rico and join the UPKM group to continue their research project as one group. The requirement for completion of this project includes two major presentations, one at UPRM and one at WPI, and one written report. The presentations are open to public, and faculty members, local industry, and parents are invited.

\section{Project Description}

The project work pursued by the student teams has involved the design of earthquake resistant structures, part of a broad civil infrastructure initiative. Specifically, the teams have sought to utilize the unique thechanical properties of shape memory alloys (SMA's) to affect structural dynamics such that damage is minimized or prevented. Initial efforts focused on using SMA's as activators to connect or discontrect bracing elements thereby deterring the structure, Presumably, detectors would sense arrival and frequency of seismic waves resulting in appropriate activation currents to be generated so that resonant response would be minimized. Student teams successfully designed SMA activated bracing elements and utilized a model building structure to demonstrate operation using an electromagnetic shaker.

More recently, project teams have employed a different strategy by utilizing the hysteretic properties of SMA's to absorb vibrational energy. SMA's loaded to a critical stress exhibit a significant reduction in effective elastic modulus because of microstructural changes. On unloading, however, the original slope is followed until the microstructural changes reverse

0-7803-5643-8/99/\$10.00 1999 IEEE thereby forming a roughly parallelepiped stress-strain curve.

The teams designed SMA structural elements such that the enclosed stress-strain area was maximized thereby maximizing energy transfer to the SMA's. The result was conversion of vibrational energy to thermal energy with dissipation through heat transfer to the atmosphere. The effectiveness of this strategy was demonstrated using a model structure with variations of SMA devices.

\section{Results}

The technical results of the collaborative research have been good. The teams have demonstrated, using model structures, the effectiveness of employing SMA's to control stnuctural vibration. They have, thus, laid the ground work for use of such techniques to mitigate earthquake damage to structures.

The student teams have necessarily made accommodation to cultural differences and thus experienced, first-hand, issues associated with global professional practice. It does take substantial effott to achieve cultural understanding between the student teams. The project advisors frequently must actively encourage teamwork and find ways to effect understanding.

The final reports, written by the combined team, reflect the learning and accomplishments resulting from the collaboration as well as the technical accomplishments. From one point of view, the development of the report is the capstone team building activity and cements the cross-cultural relationships built during the project. Delivery of oral teports at UPRM and at WPI give the project visibility and importance in the larger communities.

\section{Conclusions}

Collaborative research projects provide opportunity for both WPI and UPRM students to gain a knowledge about not only the topic of their research, but, also, about each other's culture. They learn how to work in teams, how to solve problems and how to respect an unfamiliar culture. The success of this program is evident from its results which have been presented at CANCAM 97 and $12^{\text {th }}$ ASCE Conference. As a direct result of this project an international patent has been filed for one of the devices invented during the conduct of these projects.

\section{Acknowledgments}

The project is suppor-ted by UPRM, WPI, Center for Loss Prevention and Structural Integrity, and United Technology. The supports are in terms of space, administrator help, and funding.

November 10 - 13, 1999 San Juan, Puerto Rico 